Anaesthesist $2011 \cdot 60: 743-750$

DOI 10.1007/s00101-011-1918-8

Online publiziert: 6. Juli 2011

(c) Springer-Verlag 2011

Redaktion

A. E. Goetz, Hamburg

M. Jöhr, Luzern

T. Koch, Dresden

C. Werner, Mainz

A. Zutter ${ }^{1}$. F.J. Frei ${ }^{2}$

${ }^{1}$ Abteilung für Pädiatrische Intensivbehandlung (APIB),

Universitätsklinik für Kinderheilkunde, Inselspital, Bern

${ }^{2}$ Abteilung Anästhesie, Universitäts-Kinderspital beider Basel (UKBB), Basel

\title{
Unkooperatives Kind bei Narkoseeinleitung
}

\section{Theorie und Praxis}

\begin{abstract}
Aus der Literatur sind keine Daten zur Häufigkeit von erschwerten Anästhesieeinleitungsbedingungen wegen fehlender Kooperation verfügbar. Bei den folgenden Angaben handelt es sich deshalb um Schätzungen. Bei Kindern im Vorschulalter ist das kurzfristige Festhalten nach Aufsetzen der Beatmungsmaske bzw. zum Legen eines i.v.-Zugangs wahrscheinlich in mehr als der Hälfte der Patienten notwendig. Deutlich seltener tritt fehlende Kooperation bei Kindern im Schulalter auf. Meistens kann in diesen Fällen mithilfe der hier beschriebenen Maßnahmen eine akzeptable Lösung gefunden werden. Kinder im Schulalter, die sich einer Anästhesieeinleitung völlig verweigern und bei denen ein Verschieben des Eingriffs oder die Anwendung von Zwang notwendig ist, trifft der Kinderanästhesist wahrscheinlich in 1-2\% aller Anästhesien an. Besonders anspruchsvolle Patientengruppen stellen Kinder mit geistiger Retardierung, Sprachbarrieren und solche mit wiederholten oder längeren Krankenhausaufenthalten (z. B. onkologische Patienten) dar.
\end{abstract}

Es klagen $40-60 \%$ der Kinder über perioperative Angst und Stress [1]. Kinderanästhesisten sind daher nicht selten mit wenig oder gar nicht kooperativen Patienten konfrontiert, bei denen eine zufriedenstellende Anästhesieeinleitung erschwert ist. Die Kooperation ist abhängig vom
Alter, dem geistigen Entwicklungsstand und den Charaktereigenschaften des Kindes, der Angst vor der Trennung von den Eltern, der fremden Umgebung, vor Anästhesie und Chirurgie im Allgemeinen und vor Schmerz, körperlichem Unbehagen sowie Kontroll- bzw. Autonomieverlust im Speziellen.

Im Folgenden wird nach einigen Vorbemerkungen zu quantitativen Beurteilungstests von Angst und Stress bei Kindern und Eltern sowie zur "normalen“ psychischen Entwicklung im Kindes- und Adoleszentenalter auf den Stellenwert der elterlichen Anwesenheit bei Narkoseeinleitung, verschiedener nichtpharmakologischer Maßnahmen und einer sedativen Prämedikation zur Verbesserung der Kooperation eingegangen. Mögliche Strategien beim - trotz altersgerechter Aufklärung von Patient und Eltern, einfühlsamer Zuwendung und optimaler Prämedikation - weiterhin unkooperativen Kind sind Verschiebung oder Absage des Eingriffs oder - als Mittel der letzten Wahl eine Einleitung unter Anwendung körperlichen Zwangs. Diesbezüglich soll auf einige wesentliche juristische und ethische Aspekte eingegangen werden. Schließlich wird anhand zweier Szenarien das mögliche Vorgehen beschrieben.

\section{Beurteilung von Angst, Temperament und postoperativem Verhalten}

In der Literatur gibt es eine Vielzahl verschiedener Tests und Scores zur Beurtei- lung der präoperativen Angst und des postoperativen Verhaltens. Die meisten sind personal- sowie zeitaufwendig und daher weniger für den klinischen Alltag als für wissenschaftliche Studien geeignet. Im Folgenden werden einige Scoring-Systeme beschrieben, auf die später Bezug genommen wird.

Yale Anxiety Score (YAS) und „modified Yale Anxiety Score" (mYAS). Die Tests wurden spezifisch zur Fremdeinschätzung der Angst bei Narkoseeinleitung bei Kindern entwickelt. Beurteilt werden 22 Kriterien in 5 Kategorien (Aktivität, Gesichtsausdruck, Aufmerksamkeit/ Weckbarkeit, Vokalisation und Interaktion mit Erwachsenen, [2]).

State Trait Anxiety Inventory (STAI). Dieser Test ist der Goldstandard zur Quantifizierung der Angst bei Erwachsenen. Er kann verwendet werden, um die situative Angst der Eltern direkt nach Narkoseeinleitung des Kindes zu quantifizieren [2].

Emotionality Activity Sociability Impulsivity (EASI). Der Test dient zur Beurteilung des kindlichen Temperamentes. Erhoben wird die elterliche Einschätzung von insgesamt 20 Eigenschaften in den genannten 4 Kategorien (Emotionalität, Aktivität, Geselligkeit/Ungezwungenheit, Impulsivität) auf einer Skala von 1-5 (total 20-10o Punkte, je höher, desto temperamentvoller, [2]). 
Visual Analog Scale (VAS). Analog zur Schmerzbeurteilung kann der VAS auch zur Selbst- und Fremdeinschätzung der Angst der Eltern bzw. der Kinder eingesetzt werden [2].

Posthospitalization Behaviour Questionnaire (PHBQ). Dies ist der einzige Test zur Beurteilung des kindlichen Verhaltens nach Krankenhausentlassung. Es ist ein Fragebogen für Eltern zur Einschätzung des Verhaltens ihres Kindes mit 27 Kriterien in 6 Kategorien (allgemeine Ängstlichkeit, Trennungsangst, Schlafangst, Essstörungen, Aggression gegen Autorität, Apathie/Verweigerung). Für jedes Kriterium soll das Verhalten mit demjenigen vor der Hospitalisation verglichen werden [2].

\section{Assoziation zwischen Angst und postoperativen Verhaltensänderungen}

Der Zusammenhang zwischen präoperativer Angst und negativen postoperativen Verhaltensänderungen wurde in 2 Studien von Kain et al. [3, 4] untersucht: Bei 91 Kindern im Alter von 1 bis 7 Jahren mit tageschirurgischen Eingriffen, Einleitung ohne Prämedikation und elterliche Anwesenheit war das Ausmaß der präoperativen Angst ein unabhängiger Prädiktor für das Auftreten negativer postoperativer Verhaltensänderungen. Die Häufigkeit dieser Verhaltensänderungen nahm im Laufe der Zeit ab. Kain et al. stellten Verhaltensauffälligkeiten bei $67 \%$ der Kinder am 1. postoperativen Tag, bei $45 \%$ am 2. postoperativen Tag und bei $23 \%$ am 3 . postoperativen Tag fest. Diese persistierten bis maximal 6 Monate bei $20 \%$ und bis zu einem Jahr bei 7,3\% der Kinder.

Mithilfe des PHBQ erfasste häufige Verhaltensänderungen waren schlechte Träume, nächtliches Weinen, mangelnde Folgsamkeit gegenüber Eltern, Trennungsangst und Stimmungsschwankungen [3]. Schwerwiegende Veränderungen wie neu aufgetretene Enurese waren selten (o,8\% nach 2 Wochen, [4]). Eltern, pädiatrisch und operativ tätige Kollegen und sogar Anästhesisten machen häufig die Anästhesie für diese Veränderungen verantwortlich. Dieser bequemen, aber vereinfachten Betrachtungsweise muss ent- gegengehalten werden, dass der perioperativ durchgemachte Stress, die Verletzung der körperlichen Integrität und der Aufenthalt in einer völlig fremden und z. T. für das Kind bedrohlichen Umgebung ursächlich wohl mehr ins Gewicht fallen als die Anästhesie per se. Ein wissenschaftlicher Beweis hierfür (sowie auch für die Anästhesie als alleinige Ursache) steht jedoch aus.

\section{Faktoren, die Angst und Verhalten beeinflussen}

\section{Alter}

Studien zum Einfluss des Alters auf die Inzidenz von Angst bei Narkoseeinleitung haben widersprüchliche Resultate ergeben $[2,5,6]$. Negative postoperative Verhaltensänderungen scheinen bei jüngeren Kindern häufiger zu sein (Vorschulalter, $[4,7])$. Es gilt jedoch, die altersspezifische psychologische Entwicklung zu berücksichtigen [1]. Dabei bestehen natürlich große interindividuelle Unterschiede. Die Altersangaben sind daher nur als Richtwerte zu verstehen.

In den ersten Lebensmonaten lassen sich Säuglinge auch von Fremden beruhigen. Im Alter von 6 bis 9 Monaten beginnt in der Regel die Trennungsangst („Fremdeln“). Kleinkinder (1 bis 3 Jahre) haben i. Allg. eine enge Bindung zu ihren Eltern und empfinden Trennungsangst. Das (Sprach-)Verständnis ist oft deutlich ausgeprägter als die verbalen Expressionsmöglichkeiten. Auch sind sie fähig, Traurigkeit und Angst ihrer Eltern wahrzunehmen. Sie sind zu jung für situative Erklärungen, aber sind oft ablenkbar und (auch durch Fremde) zu beruhigen. Im Vorschulalter ( 4 bis 6 Jahre) fordern viele Kinder Erklärungen und möchten das Gefühl der Kontrolle über die Situation, z. B. durch Verweigerung. Schulkinder (7 bis 12 Jahre) haben häufig konkrete Ängste in Bezug auf die bevorstehende Narkose („nicht mehr aufwachen“) bzw. Operation (Schmerzen, Verstümmelung). Sie möchten und sollten, wenn möglich, in Entscheidungen eingebunden werden ( $\mathrm{z}$. B. inhalative oder i.v.-Einleitung), um ein Gefühl der Kontrolle und Autonomie zu bekommen. Im Adoleszentenalter müssen Jugendliche lernen, selber Entscheidun- gen zu treffen. Die Suche nach Unabhängigkeit kann zu Machtkämpfen mit Eltern und Ärzten als Autoritätspersonen führen. Die Angst vor Gesichts- oder Kontrollverlust kann sehr ausgeprägt sein. Insbesondere gilt es, die Intimsphäre zu respektieren (z. B. Zugedecktlassen, Ausziehen von Unterwäsche erst nach Narkosebeginn).

Besonders anspruchsvoll sind, unabhängig vom Alter, Patientengruppen mit Verständigungsschwierigkeiten bzw. limitierten Kommunikationsmöglichkeiten, mental retardierte Kinder und Patienten mit Sprachbarrieren.

\section{Geschlecht}

Das Geschlecht beeinflusst weder die Inzidenz präoperativer Angst noch das Auftreten postoperativer Verhaltensänderungen $[4,7]$.

\section{Temperament}

Scheue, gehemmte Kinder (niedriger EASI Score für Aktivität) haben mehr Angst in der präoperativen Wartezone und bei der Trennung von den Eltern [4]. Impulsive Kinder (hoher EASI Score für Impulsivität) haben ein erhöhtes Risiko für allgemeine Angst und negative postoperative Verhaltensänderungen.

\section{Frühere Krankenhauserfahrungen}

In mehrere Studien waren Kinder mit früheren negativen Krankenhauserfahrungen sowohl ängstlicher in der präoperativen Wartezone und bei der Trennung von den Eltern [4] als auch weniger kooperativ bei Narkoseeinleitung [7]. Besonders anspruchsvolle Patientengruppen sind also onkologische Patienten (Diagnostik, „Follow-up“-Untersuchungen) oder Kinder, bei denen regelmäßige Eingriffe notwendig sind.

\section{Art der Narkoseeinleitung}

In einer finnischen Studie [8] wurden 92 nichtmedikamentös prämedizierte Kinder im Alter von 2 bis 7 Jahren mit tageschirurgischen HNO-Eingriffen für eine i.v.-, inhalative oder rektale Anästhesieeinleitung (Methohexital) randomisiert. 
Die Kooperation bei Einleitung auf einer 5er-Skala wurde als Surrogatparameter für Angst genommen. Die Kooperation war am besten bei der inhalativen, gefolgt von der rektalen und i.v.-Einleitung [ohne „Eutectic-mixture-of-local-anesthetics“(EMLA-)Pflaster]. Am meisten negative Erinnerungen hatten Kinder an die inhalative Einleitung. Bezüglich des Auftretens von postoperativen Verhaltensänderungen bestand kein Unterschied.

\section{Art des Eingriffs}

Zwischen verschiedenen chirurgischen Disziplinen und tageschirurgischen oder stationären Eingriffen konnten bezüglich des postoperativen Verhaltens keine Unterschiede festgestellt werden [2].

\section{Interventionen zur Verminderung der kindlichen Angst}

\section{Anwesenheit der Eltern}

Aufgrund der aktuellen Studienlage verbessert die Anwesenheit der Eltern bei der Einleitung weder das präoperative Angstniveau noch die Kooperation der Kinder während der Einleitung, noch reduziert es die Inzidenz postoperativer Verhaltensprobleme [9].

In einer Studie ([10], 84 Kinder, 2 bis 6 Jahre alt) wurde neben verschiedenen Scores (u. a. EASI, STAI, VAS, YPAS), die keinen signifikanten Unterschied zwischen der Interventions- (Einleitung mit Eltern) und der Kontrollgruppe (Einleitung ohne Eltern) ergaben, der Kortisolspiegel beim Kind unmittelbar nach inhalativer Einleitung als Indikator für Stress gemessen. Drei Gruppen hatten hier einen signifikant tieferen Spiegel bei Anwesenheit der Eltern: Kinder älter als 4 Jahre, Kinder mit geringer Aktivität im EASIScore und Kinder wenig ängstlicher Eltern gemäß STAI Score. Positiv anzumerken ist, dass die Anwesenheit der Eltern in dieser Studie weder einen Einfluss auf die Sicherheit noch auf die Dauer der Einleitung, noch auf den Stress-Level des Anästhesisten (STAI, Puls- und Blutdruckmessung) hatte. Während die Mehrzahl der Eltern meinte, durch ihre Präsenz die Arbeit des Anästhesisten zu erleichtern (68\%) und ihrem Kind zu helfen (90\%), war dies in der Einschätzung des Anästhesisten nur in $31 \%$ resp. $12 \%$ der Fälle so.

Es besteht ein Zusammenhang zwischen Ängstlichkeit der Kinder und der Eltern, gemessen mit dem STAI Score. Mütter sind dabei i. Allg. ängstlicher als Väter (• Abb. 1; [11]). Weitere Prädiktoren elterlicher Angst sind Kindesalter unter 1 Jahr, wenn es sich um eine erste Operation/Anästhesie oder ein Einzelkind handelt oder die Eltern einen Beruf im Gesundheitswesen ausüben [12, 13].

In 2 Studien von Kain et al. wurde der Effekt elterlicher Anwesenheit mit demjenigen einer Prämedikation mit Midazolam verglichen. In der ersten Studie ([12], 88 Kinder, 2 bis 6 Jahre alt) waren die Kinder in der Prämedikationsgruppe (Midazolam 0,5 mg/kgKG, oral) signifikant weniger ängstlich (mYPAS) und kooperativer als die Kinder, die von einem Elternteil begleitet wurden. Deren Angst-Level lag gleich wie in der Kontrollgruppe (weder Begleitung noch Prämedikation). Interessanterweise war bei den prämedizierten Kindern auch der Angst-Level der Eltern nach der Trennung vom Kind (STAI Score) signifikant tiefer. Bezüglich postoperativer Verhaltensänderungen bestand in den 3 Gruppen kein Unterschied.

In der zweiten Studie ([14], 103 Kinder, 2 bis 8 Jahre alt), wurde der Effekt elterlicher Anwesenheit und einer oralen Prämedikation mit Midazolam (0,5 mg/ kgKG) mit demjenigen einer alleinigen Prämedikation verglichen. Hier ergab sich kein zusätzlicher Benefit durch die Anwesenheit der Eltern bezüglich kindlicher Angst (mYPAS). Die elterliche Angst nach der Trennung vom Kind war jedoch tiefer und die allgemeine Zufriedenheit sowohl mit dem Trennungsprozess als auch der anästhesiologischen, chirurgischen und pflegerischen Betreuung insgesamt höher, wenn die Eltern während der Anästhesieeinleitung anwesend waren.

Zusammengefasst profitieren allenfalls charakterlich ruhige Kinder wenig ängstlicher Eltern von der Anwesenheit der Eltern bei Einleitung. Vermutlich ist die elterliche Angst ein häufiger Grund für den mangelnden Effekt der Anwesenheit auf die kindliche Angst. Dennoch sollte, falls dies aus betrieblichen und personellen Gründen möglich ist, dem Wunsch der meisten Eltern, ihr Kind zur Einlei-
Anaesthesist $2011 \cdot 60: 743-750$

DOI 10.1007/s00101-011-1918-8

(C) Springer-Verlag 2011

\section{A. Zutter · F.J. Frei \\ Unkooperatives Kind bei Narkoseeinleitung. Theorie und Praxis}

\section{Zusammenfassung}

Perioperative Angst und daraus resultierende mangelnde Kooperation bei der Narkoseeinleitung sind ein häufiges Problem in der Kinderanästhesie. Das Ausmaß der Angst hängt von verschiedenen kindlichen, aber auch von elterlichen Faktoren, dem Anästhesieteam sowie der kinderfreundlichen Atmosphäre und Infrastruktur des Krankenhauses ab. Neben einer Prämedikation gibt es verschiedene nichtpharmakologische Maßnahmen, um die kindliche Angst zu reduzieren und damit die Narkoseeinleitung zu erleichtern. Bleibt das Kind trotz aller vorbereitenden Maßnahmen weiterhin unkooperativ, muss grundsätzlich unter Berücksichtigung verschiedener Kriterien entschieden werden, ob der Eingriff, falls medizinisch vertretbar, verschoben wird oder ob die Narkoseeinleitung unter Anwendung von körperlichem Zwang durchgeführt werden soll.

Schlüsselwörter

Behandlungsverweigerung · Angst . Prämedikation · Sedativa $\cdot$ Eltern

\section{Uncooperative children during induction of anesthesia. Theory and practice}

\section{Abstract}

Perioperative anxiety and the resulting lack of cooperation during induction of anesthesia is a common problem in pediatric anesthesia. The extent of anxiety depends on a variety of factors concerning the child, the parents, the anesthesia team and the hospital's friendly atmosphere and infrastructure. Apart from premedication there are a number of non-pharmacological means to improve the child's cooperation and thereby facilitate the induction of anesthesia. If the child is still uncooperative despite an optimal preparation, the anesthetist has to decide whether to postpone the operation or to perform induction of anesthesia under physical restraint in consideration of various criteria.

Keywords

Treatment refusal · Anxiety - Premedication . Sedatives - Parents 


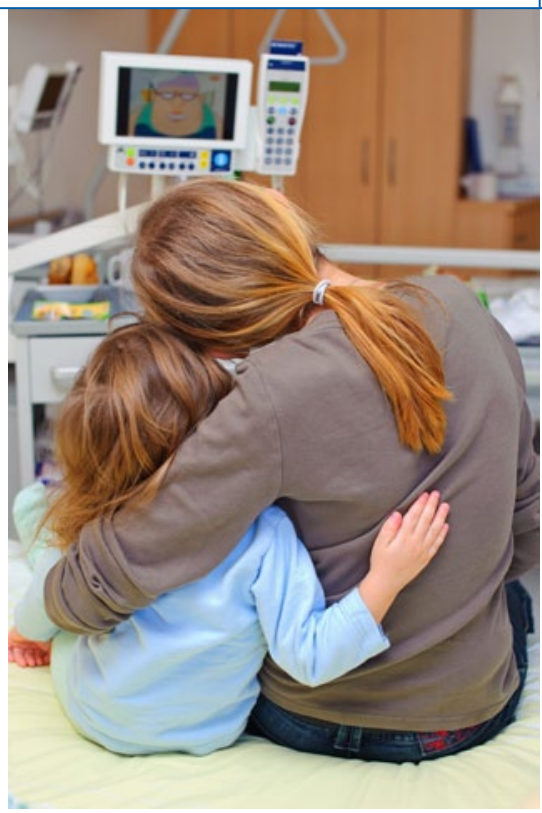

Abb. $1 \Delta$ Es besteht ein Zusammenhang zwischen Ängstlichkeit der Kinder und der Eltern; Mütter sind dabei im Allgemeinen ängstlicher als Väter

tung zu begleiten, entsprochen werden, da dies zu einer erhöhten Zufriedenheit und geringeren Ängsten nach der Trennung führt. Durch eine orale Prämedikation mit Midazolam können sowohl kindliche als auch elterliche Angst signifikant reduziert werden.

\section{Präoperative Vorbereitung und nichtpharmakologische Interventionen}

Neben der Elternpräsenz bei Einleitung werden verschiedene nichtmedikamentöse Maßnahmen routinemäßig eingesetzt und spiegeln „Common-sense“-Praxis wider [15]. Dazu zählen u. a. schriftliche Informationen und das präoperative Gespräch. Die Bedeutung des präoperativen Gesprächs ist groß, können hier doch einerseits Ängste bei Kindern und Eltern angesprochen und zumindest teilweise abgebaut werden sowie andererseits unkooperatives Verhalten in manchen Fällen antizipiert und entsprechend notwendige Maßnahmen mit den Eltern vorbesprochen werden.

Fortier et al. [16] untersuchten mithilfe eines insgesamt 4o Fragen umfassenden, am Operationstag verteilten Fragebogens, welche Informationen den Patienten (143 Kinder, 7 bis 17 Jahre alt)

Allgemeinanästhesie

wichtig sind. Besonders hoch gewichtet wurden von den Patienten Informationen betreffend postoperative Schmerzen und zudem bei den präadoleszenten Kindern ( 7 bis 11 Jahre alt) Fragen zur perioperativen Umgebung (Operationssaal, Aufwachraum).

Aufwendigere Vorbereitungsprogramme (Informationsvideo, Führung in die Operationsabteilung, Rollenspiele) brachten in Studien keinen allgemeinen Nutzen. Einzelne Patientengruppen (Kinder mit früheren Hospitalisationen, mit hoher Emotionalität im EASI Score und Kinder im Alter von 2 bis 3 Jahren) waren in einer Studie [17] sogar ängstlicher als vor dem Vorbereitungsprogramm. Dieselbe Studie ergab, dass der optimale Zeitpunkt für eine solche Vorbereitung je nach Alter variiert ( 1 bis 2 Tage vor dem Eingriff für 2bis 4-Jährige, 5 bis 10 Tage vor dem Eingriff für 5- bis 12-Jährige), was die Umsetzung im Alltag zusätzlich erschwert.

In einer 2009 publizierten, 17 Studien umfassenden Cochrane-Datenanalyse [18] wurden neben Elternpräsenz verschiedene andere nichtpharmakologische Interventionen am Tag der Operation bzw. Anästhesie untersucht. Dabei hatten spezifische Maßnahmen wie Ohrakupunktur bei den Eltern [19], Präsenz von Krankenhaus-Clowns während der Einleitung [20], Hypnose [21], Videospiele [22] und eine ruhige Umgebung einen angstvermindernden Effekt. Daneben wurde auch schon der erfolgreiche Einsatz des iPhone als Hilfsmittel bei der Narkoseeinleitung anekdotisch beschrieben [23]. Das Problem dieser Maßnahmen besteht - außer der für jede Einleitung zu schaffenden möglichst ruhigen Umgebung - im zusätzlichen personellen und zeitlichen Aufwand.

\section{Pharmakologische Prämedikation}

Das „ideale Anästhesieeinleitungsmedikament" wäre ein geschmackloser Wirkstoff, der in flüssiger Form vorliegt, vom Kind zusammen mit einem gewohnten Getränk eingenommen werden kann und zu einer kurz dauernden tiefen Sedation bzw. leichten Anästhesie führt. Die eigentliche Anästhesieeinleitung würde dann inhalativ oder i.v. erfolgen. Ein solches Medikament existiert nicht; es soll deshalb in der Folge kurz auf die am häufigsten zur Prämedikation verwendeten Medikamente eingegangen und ein möglicher Stufenplan aufgezeigt werden [9, 24, 25].

\section{Midazolam}

Das mit Abstand am häufigsten eingesetzte Medikament zur Prämedikation von Kindern ist das rasch und kurz wirksame Benzodiazepin Midazolam. Es kann oral, rektal, nasal, i.v. oder i.m. appliziert werden. Bei Säuglingen ab 6 Monaten und je nach Präferenz bis ins Vorschulalter (sowie ggf. auch bei älteren, geistig Behinderten) steht die rektale, bei den übrigen Kindern die orale Verabreichung im Vordergrund. Lehrbücher empfehlen für beide Applikationswege Dosierungen von $0,5-1 \mathrm{mg} / \mathrm{kgKG}$ mit einer Maximaldosis von $15 \mathrm{mg}$. Bei oraler Verabreichung hat sich bereits eine Dosierung von 0,25 mg/kgKG als effektiv erwiesen [26]; bei Dosierungen über 0,5 mg/kgKG kann sich die postoperative Erholungszeit verlängern.

Das „timing“ der Prämedikation ist entscheidend. Sedation und anterograde Amnesie treten nach etwa 10 min, der anxiolytische Effekt tritt nach etwa 15 min ein und erreicht nach 20-30 min das Maximum. Der sedative Effekt klingt nach 45-6o min wieder ab.

Wie bei Erwachsenen beeinflusst Midazolam v. a. das explizite Gedächtnis, während diffuse implizite Erinnerungen an unangenehme Ereignisse während der Einleitung erhalten bleiben können [27]. Dies erklärt möglicherweise die widersprüchlichen Daten bezüglich des Einflusses auf das Auftreten postoperativer Verhaltensveränderungen. So fanden Kain et al. [28] eine Woche postoperativ signifikant weniger mithilfe des PHBQ erfasste negative Verhaltensänderungen in der mit Midazolam prämedizierten Gruppe, verglichen mit der Placebogruppe. Am ausgeprägtesten waren die Unterschiede bezüglich Essstörungen und Trennungsangst. Zwei Wochen postoperativ bestand kein signifikanter Unterschied mehr zwischen den beiden Gruppen. Cox et al. [29] konnten bei einer Sichtung der Literatur von 1990-2006 keinen konsistenten Effekt einer Prämediaktion mit Midazolam auf das postoperative Verhalten feststellen. In der Studie von Calipel et al. [21], 
die den Effekt von Hypnose und Midazolam miteinander verglich, waren Verhaltensauffälligkeiten in der Hypnosegruppe am 1. (30 vs. 62\%) und 7. postoperativen Tag ( 26 vs. $59 \%$ ) deutlich seltener. Fehlendes Ansprechen auf orales Midazolam ist in $10-20 \%$ der Fälle beschrieben. Der Hauptgrund liegt vermutlich in der extrem variierenden Bioverfügbarkeit (971\%, durchschnittlich 36\%). Paradoxe Reaktionen sind bei Kindern selten.

Die nasale Gabe verursacht wegen des niedrigen $\mathrm{pH}$ ein unangenehmes Brennen in den Nasenhöhlen und scheint daher für Kinder eher ungeeignet. Der Vorteil der i.v.-Gabe (gemäß Schweizerischem Arzneimittelkompendium 2011: 6 Monate bis 5 Jahre: $0,05-0,1 \mathrm{mg} / \mathrm{kgKG}$, total $<6 \mathrm{mg}$; 6 bis 12 Jahre: $0,025-0,05 \mathrm{mg} / \mathrm{kgKG}$, total $<10 \mathrm{mg}$ ) besteht im raschen Wirkungseintritt innerhalb von 2-3 min. Die Autoren empfehlen ein Eintitrieren bis zum gewünschten Effekt. Wegen der potenziellen Gefahr einer Atemdepression sollte die i.v.-Applikation jedoch nur beim monitorisierten Kind und bei Verfügbarkeit von Personal, das mit dem Management atemdeprimierter Patienten vertraut ist, erfolgen. Bei einer Überdosierung besteht die Möglichkeit einer Antagonisierung mit i.v.-verabreichtem Flumazenil (o,o1 mg/kgKG).

\section{Ketamin}

Eine gute Alternative zu Midazolam stellt der N-Methyl-D-Aspartat-Glutamat(NMDA)-Rezeptor-Antagonist Ketamin [30] oder dessen S(+)Isomer S-Ketamin dar [31]. Die Applikation ist oral, rektal, i.v. oder i.m. möglich. Ein Vorteil von Ketamin als Prämedikation ist der zusätzliche analgetische Effekt.

Die orale Dosierung liegt bei 3-6 mg/ kgKG. Der sedative Effekt (dissoziative Anästhesie) tritt nach 20-30 min ein. Bei oraler Gabe liegt die Bioverfügbarkeit wegen des hohen hepatischen „First-pass“Effekts bei lediglich 16\%. Eine Atemdepression ist bei genannter Dosierung nicht zu befürchten. Allerdings treten unangenehme Nebenwirkungen bei einer Dosierung von 3-6 mg/kgKG relativ häufig auf (Hypersalivation 13-33\%, Nystagmus 33$60 \%$ [32]) mit der Folge, dass Ketamin allein in der Routine nur selten oral oder rektal eingesetzt wird.
Warner et al. [33] verglichen in einer Studie (6o Kinder, 1,5 bis 8 Jahre alt) den Effekt einer peroralen Prämedikation mit 0,5 mg/kgKG Midazolam (Gruppe 1), $6 \mathrm{mg} / \mathrm{kgKG} \mathrm{Ketamin} \mathrm{(Gruppe} \mathrm{2)} \mathrm{so-}$ wie $0,4 \mathrm{mg} / \mathrm{kgKG}$ Midazolam und $4 \mathrm{mg} /$ kgKG Ketamin (Gruppe 3), jeweils kombiniert mit o,02 mg/kgKG Atropin 2030 min vor Einleitung. Der Sedationsgrad wurde bei der Trennung von den Eltern und der Maskeneinleitung auf einer 5er-Skala beurteilt (1: schwierig weckbar, 2: leicht weckbar, 3: wach, ruhig, 4: wach, ängstlich, 5: weinend, agitiert); hierbei wurden Grad 1-3 als erfolgreiche Prämedikation bezeichnet. Die Erfolgsquote betrug bei der Trennung von den Eltern 75\% für Midazolam, 90\% für Ketamin und $100 \%$ für die Kombination, für die Maskeneinleitung 42\% mit Ketamin, 65\% mit Midazolam und $85 \%$ für die Kombination. Verschiedene Institutionen verwenden mit Erfolg dieselbe Medikamentenkombination zur rektalen Prämedikation bzw. Narkoseeinleitung [34]. An der eigenen Klinik haben die Autoren keine Erfahrung mit dieser kombinierten peroralen oder rektalen Prämedikation.

Beim agitierten unkooperativen $\mathrm{Pa}$ tienten besteht die Möglichkeit einer i.m.-Gabe in den Oberarm (M. deltoideus) oder alternativ in den Oberschenkel (M. quadriceps). Mit einer Dosierung von $3-5 \mathrm{mg} / \mathrm{kgKG}$ Ketamin kann innerhalb von 3-4 min eine effektive Sedation erreicht werden. Nach i.v.-Gabe von 1-2 mg/kgKG Ketamin tritt die Wirkung bereits nach 1-2 min ein. Die Wirkungsdauer liegt bei etwa $45 \mathrm{~min}$.

\section{Clonidin}

Eine weitere Möglichkeit ist die Gabe des $\alpha_{2}$-Agonisten Clonidin [35] in einer Dosierung von $2-4 \mu \mathrm{g} / \mathrm{kgKG}$ oral oder 4-5 $\mu \mathrm{g} / \mathrm{kgKG}$ rektal. Ursprünglich als Antihypertensivum entwickelt, besitzt Clonidin einen anxiolytischen, analgetischen (opiatsparenden) und sedativen Effekt. Im Gegensatz zu Midazolam ergibt sich weder eine psychotrope noch eine amnestische Wirkung. Die Patienten sind „normal“ schläfrig und weckbar. Eine Sedation tritt allerdings erst 60-90 min nach peroraler Gabe ein und hält bis $24 \mathrm{~h}$ an. Dies erschwert einerseits den Einsatz im ambulanten Bereich, kann

\section{Hier steht eine Anzeig}

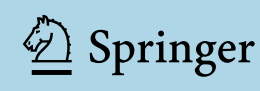


andererseits im stationären Bereich sogar von Vorteil sein.

Bei Erwachsenen reduziert Clonidin die Inzidenz von postoperativem „shivering “. Bei Kindern bestehen Hinweise, dass es nach Sevoflurananästhesien und zusätzlicher i.v.-, kaudaler und rektaler Clonidingabe seltener zu postoperativer Agitation kommt. Obwohl in der Literatur keine Fälle schwerwiegender Hypotensionen oder Bradykardien beschrieben sind, benutzen Bergendahl et al. [35] sowohl für die orale als auch für die rektale Prämedikation eine Mischung aus $4 \mu \mathrm{g} / \mathrm{kgKG}$ Clonidin und $20 \mu \mathrm{g} / \mathrm{kgKG}$ Atropin. Kontraindikationen für Clonidin sind Hypovolämie und atrioventrikuläre (AV-)Blockierungen.

\section{Thiopental, rektal}

Eine etwas in Vergessenheit geratene Option ist die rektale Applikation von Thiopental $30-40 \mathrm{mg} / \mathrm{kgKG}$ in einer speziell angefertigten 10\%igen Lösung. Dabei tritt innerhalb von 6-10 min eine tiefe Sedation ein. Diese Methode kommt bei den Autoren nur noch in Ausnahmefällen zum Einsatz, z. B. bei geistig retardierten Patienten, bei denen eine perorale Medikamentengabe nicht möglich ist, oder bei Kindern, bei denen mit hoher Wahrscheinlichkeit fehlende Kooperation zu erwarten ist. Da es sich bei dieser Methode eigentlich um eine Anästhesieeinleitung handelt, sollte eine Anästhesiefachperson unmittelbar nach der Verabreichung anwesend sein. Ein Nachteil der Methode ist die verlängerte 2-3 $\mathrm{h}$ dauernde Aufwachzeit, die eine ambulante Betreuung erschwert oder sogar verbietet.

\section{Topische Lokalanästhesie (EMLA ${ }^{\circledR}$ ) bei geplanter i.v.-Einleitung}

Bei geplanter i.v.-Einleitung empfiehlt sich in der Regel eine Vorbereitung der potenziellen Einstichstellen mit topischer Lokalanästhesie (z. B. EMLA-Creme) zur Verminderung des Punktionsschmerzes. Die Creme sollte mindestens 45-60 min einwirken können und mit Vorteil 15 min vor Punktion wieder entfernt werden, damit die Vasokonstriktion etwas abklingen kann. Bei Säuglingen mit viel subkutanem Fettgewebe oder Patienten mit schwierigen Venenverhältnissen kann EMLA-Creme die Punktionsbe- dingungen weiter erschweren. Dies sollte im Prämedikationsgespräch thematisiert werden (mehr Punktionsschmerz vs. Mehrfachpunktionen).

\section{Vorgehen in der Praxis}

\section{„Extremlösungen"}

Wie aber weiter, wenn ein Kind trotz altersgerechter Aufklärung, einfühlsamer Zuwendung und optimaler Prämedikation weiterhin unkooperativ ist? Hier bieten sich zwei „Extremlösungen“ an:

- Verschiebung bzw. Absage des Eingriffs,

- als Ultima Ratio Einleitung unter Anwendung körperlichen Zwangs („physical restraint").

Eine 2007 publizierte Umfrage bestehend aus einem strukturierten Fragenbogen und hypothetischen Fallbeispielen [36] bei Mitgliedern der US-amerikanischen Society of Pediatric Anesthesiologists $(n=453 / 852$, entsprechend einer Rücklaufquote von 57\%) ergab folgende Ergebnisse: Es hatten 9\% der Teilenehmer im Laufe des letzten Jahres, $25 \%$ im Laufe der letzten 5 Jahre und $45 \%$ im Laufe ihrer gesamten Karriere mindestens einen Eingriff wegen Verweigerung/fehlenden Einverständnisses des Kindes abgesagt. Bei den folgenden Fragen wurde zwischen $4 \mathrm{Al}$ tersgruppen unterschieden. Die Frage, ob sie bei einer Mehrzahl (>50\%) der Fälle in der jeweiligen Altersgruppe bei der Einleitung körperlichen Zwang anwenden würden, bejahten für die <1-Jährigen $44 \%$, für die 1- bis 4-Jährigen $32 \%$, für die 5- bis 11-Jährigen $10 \%$ und für die $>12$-Jährigen $2 \%$ der Kinderanästhesisten. Das dabei bestehende ungute Gefühl nahm mit zunehmendem Patientenalter zu (3, 4, 9 resp. $13 \%$ für die 4 Alterskategorien). Das mediane Alter, bei dem die Antwortenden im Allgemeinen eine Ablehnung des Eingriffs durch das Kind respektieren würden, lag bei 12 Jahren.

\section{Ethische und juristische Überlegungen}

Die Berücksichtigung von ethisch/juristischen und praktischen Faktoren erleichtert die Entscheidung, ob ein Verschieben des Eingriffs oder die Anwendung von körperlichem Zwang gewählt werden soll.

Juristisch gesehen muss vor jedem Eingriff eine Einverständniserklärung vom Patienten bzw. seinem Vertreter eingeholt werden. Patienten sind ab dem 18. Altersjahr mündig und können selber ihr Einverständnis („informed consent“) geben. Unter 18 Jahren werden Kinder meistens durch ihre Eltern vertreten. Ethisch betrachtet sind aber gesunde Kinder bereits vorher urteilsfähig und damit in der Lage, ihr Einverständnis für einen geplanten Eingriff zu geben (im Englischen als ,assent", Billigung, bezeichnet) oder abzulehnen $[37,38]$.

Die publizierten ethisch-medizinischen Richtlinien der Schweizerischen Akademie der Wissenschaften [39] betonen, dass grundsätzlich immer Urteilsfähigkeit vermutet werden sollte. Folgende Fähigkeiten spielen dabei eine Rolle:

a) Die Information kann in Bezug auf die $\mathrm{zu}$ fällende Entscheidung verstanden werden.

b) Situation und Konsequenzen, die sich aus alternativen Möglichkeiten ergeben, können richtig abgewogen werden.

c) Die erhaltene Information kann im Kontext eines kohärenten Wertsystems rational gewichtet werden.

d) Die eigene Wahl kann geäußert werden.

Auch einem 10-jährigen Kind muss eine gewisse Urteilsfähigkeit attestiert werden. Es obliegt der Verantwortung des Anästhesisten, nach einem Gespräch mit den Mitbeteiligten die Urteilsfähigkeit abzuschätzen und im Sinne des Patienten zu entscheiden. Voraussetzung hierzu sind ruhige, dem Alter des Kindes angemessene Erklärungen in einem möglichst stressfreien Umfeld.

In der 1998 von der World Medical Association verabschiedeten Deklaration von Ottawa zum Recht des Kindes auf gesundheitliche Versorgung [40] steht unter Punkt 9 zum Thema Zustimmung und Selbstbestimmung: „Das Kind und seine Eltern bzw. gesetzlichen Vertreter haben das Recht, über alle Entscheidungen in Bezug auf die gesundheitliche Versorgung des Kindes informiert und aktiv daran beteiligt zu werden. In diesen Ent- 
scheidungsprozessen sollten die Wünsche des Kindes berücksichtigt werden und in Abhängigkeit von der geistigen Reife des Kindes zunehmende Bedeutung erhalten. Das nach ärztlichem Urteil entscheidungsfähige Kind hat das Recht, die Entscheidungen über seine gesundheitliche Versorgung selbst zu treffen.",

Insbesondere, wenn Konflikte bezüglich des Eingriffs zwischen den beiden Eltern oder zwischen Eltern und Kind vorhanden sind, empfiehlt es sich, den Eingriff erst nach Klärung des Konflikts zu einem späteren Zeitpunkt durchzuführen, sofern dies in Anbetracht der Dringlichkeit des Eingriffs möglich ist. Eventuell müssen dabei Fachpersonen (Hausarzt, andere beratende Personen, Vormundschaftsbehörde etc.) hinzugezogen werden.

\section{Praktische Aspekte}

Das Problem der mangelnden Kooperation bzw. Einwilligung kann sich in ganz unterschiedlichen Situationen und Zeitpunkten stellen. Das Prozedere hängt entscheidend von der Notwendigkeit und Dringlichkeit des geplanten Eingriffs ab.

Idealerweise kann eine mangelnde Kooperation antizipiert werden (Eindruck während des präoperativen Gesprächs, Erfahrungen anlässlich früherer Eingriffe), und verschiedene Strategien können besprochen werden (z. B. höher dosierte, andere oder kombinierte Prämedikation als bei früherem Eingriff, Festhalten für Maskeneinleitung, i.m.-Ketamin-Gabe, Absage des Eingriffs).

Schwieriger sind unerwartete Situationen, in denen das Kind die Einnahme der Prämedikation verweigert oder diese ungenügend wirkt. Hier besteht oft auch Zeitdruck, der den Anästhesisten zu raschen Entscheidungen drängt. Zum Abschluss soll nun das mögliche Vorgehen beim unkooperativen Kind anhand von zwei Fallszenarien besprochen werden.

\section{Szenario 1}

Bei dem 8-jährigen übergewichtigen türkischstämmigen Mehmet, der in der Schweiz geboren wurde und gut deutsch spricht, ist eine Tonsillektomie geplant. Während der Voruntersuchung verwei- gert Mehmet das Gespräch und lässt sich auch nicht untersuchen. Laut der Mutter habe er anlässlich der Zahnsanierung vor 4 Jahren, seiner bisher einzigen Vollnarkose, sehr gut auf Midazolamsirup angesprochen, sodass eine problemlose Maskeneinleitung durchgeführt werden konnte. Geplant wird eine Prämedikation mit 15 mg Midazolamsaft bei einem Körpergewicht von $50 \mathrm{~kg}$ mit anschließender inhalativer Einleitung im Beisein der Mutter. Am Operationstag weigert sich Mehmet, seine Kleidung auszuziehen und die Prämedikation einzunehmen. Die Pflegefachfrau kontaktiert nun den Anästhesiearzt und beschreibt die Situation.

Vorgehen. Der Anästhesist bittet die Pflegefachfrau, die Mutter in den OP-Bereich zu begleiten. In der Zwischenzeit vergewissert er sich beim Operateur, dass der Eingriff, wenn auch nicht dringlich, so doch absolut notwendig sei. Die Situation wird mit der Mutter im Elternbesprechungsraum in Abwesenheit des Kindes in Ruhe erörtert. Die Mutter, die sehr gut deutsch spricht, erklärt, dass der Eingriff nun schon mehrmals verschoben wurde und sie und ihr Mann eine Woche Ferien geplant hätten, um das Kind optimal betreuen zu können. Ihr Mann, der Neurologe ist, und sie selber bitten den Anästhesiearzt, dass der Eingriff nun durchgeführt werde. Auf die vorgeschlagene Alternative, dass dieser verschoben und erst nach Gesprächen mit Drittpersonen durchgeführt werden könnte, geht die Mutter nicht ein. Man einigt sich, dass der Junge nach entsprechender Information auf der Abteilung eine i.m.-Injektion erhalten solle. Daraufhin werden dem sich heftig wehrenden Patienten 150 mg Ketamin i.m. verabreicht. Nach Wirkungseintritt des Medikaments wird der Patient in den OP-Bereich begleitet und die Anästhesie weitergeführt.

Kommentar. Für den Anästhesisten bestand hier die Möglichkeit, den Eingriff zu verschieben. Da die Argumentation der Mutter während des 15 min dauernden Gesprächs jedoch nachvollziehbar war und eine Änderung der ablehnenden Haltung des Kindes nach Verschieben des Eingriffs als unwahrscheinlich erachtet wurde, hat der Anästhesist mit ungutem Gefühl entschieden, die Anästhe- sie gegen den Willen des Jungen durchzuführen. Dabei erschien eine i.m.-Ketamin-Gabe bei dem sich völlig verweigernden Kind als das kleinere Übel gegenüber einem traumatisierenden Einleitungsversuch (inhalativ oder i.v.) unter körperlichem Zwang.

\section{Szenario 2}

Bei der 5-jährigen Lara sind für den folgenden Tag eine Adenotomie und Tonsillektomie geplant. Lara ist $16 \mathrm{~kg}$ schwer und war außer rezidivierender, zuletzt monatlich auftretender Tonsillitiden bisher gesund. Anlässlich der anästhesiologischen Vorbesprechung ist sie sehr ängstlich, redet nicht, versteckt sich hinter dem Rücken der Mutter und lässt sich nicht untersuchen. Geplant werden eine orale Prämedikation mit $8 \mathrm{mg}$ Midazolam 20 min vor Anästhesiebeginn und eine inhalative Einleitung mit Sevofluran in Anwesenheit der Mutter. Es wird besprochen, dass alternativ auch eine rektale Prämedikation möglich wäre und dass je nach Wirkung der Prämedikation Lara bei der Maskeneinleitung mithilfe der Mutter „etwas festgehalten werden" müsse.

Am Operationstag nimmt Lara den Midazolamsirup unter Aufsicht problemlos ein und wird $25 \mathrm{~min}$ später in den OP-Trakt bestellt. Vor dem Betreten des Anästhesieeinleitungsraums klammert sie sich weinend an ihre Mutter, die vorwurfsvoll sagt, das Medikament habe gar nicht gewirkt.

Vorgehen. Es wird der Mutter empfohlen, das Kind auf ihren Schoß zu setzen und seine Arme sanft festzuhalten, damit die Maskenanästhesie begonnen werden könne. Die Mutter ist mit diesem Vorgehen einverstanden, und der Anästhesiearzt setzt die Maske unter Festhalten des Kopfes dicht auf Mund und Nase. Nach kurzer Zeit wehrt sich das Kind nicht mehr, es wird auf den OP-Tisch gelegt, das Monitoring wird angelegt und die Anästhesie weitergeführt.

Kommentar. Trotz adäquater Midazolamdosierung (o,5 mg/kgKG p.o.) und gutem Timing der Gabe war die Wirkung der Prämedikation ungenügend. Lara war bereits anlässlich des Prämedikationsgesprächs am Vortag sehr verängstigt. Lara ist in ihrem Alter und Zu- 
stand sicherlich nicht urteilsfähig, sodass das weitere Prozedere mit der Mutter allein festgelegt werden musste. Eine ruhige differenzierte Diskussion mit der Mutter war in dieser Situation nicht möglich, sodass, wie bereits im Rahmen der Vorbesprechung erwähnt, die Maskenanästhesie mit einem gewissen Zwang durchgeführt wurde. Alternativ hätte Lara auf die Abteilung zurückgeschickt und ein zweites Mal, z. B. mit einer Kombination aus Midazolam und Ketamin, prämediziert werden können. Neben der Zeitverzögerung gilt es dabei jedoch, den zusätzlichen Stress (insbesondere auch für die Mutter) und die Möglichkeit einer erneuten ungenügenden Wirkung der Prämedikation zu bedenken.

\section{Fazit für die Praxis}

\section{Mangelnde Kooperation bei der Narko- seeinleitung ist ein häufiges Problem in der Kinderanästhesie, v. a. bei Kindern im Vorschulalter. Die Anwesenheit der El- tern bei der Narkoseeinleitung wird von den meisten Eltern gewünscht, hat je- doch keinen wissenschaftlich nachweis- baren Effekt auf die Angst oder auf post- operative Verhaltensauffälligkeiten der Kinder. Die meisten Kinder profitieren von einer Prämedikation; hierbei ist Mi- dazolam das verbreitetste Medikament. Bezüglich nichtpharmakologischer Maß- nahmen (z. B. Hypnose) gibt es verschie- dene, in Einzelstudien erfolgverspre- chende Ansätze, die jedoch zeit- und per- sonalintensiv sind. Wenn immer medizi- nisch vertretbar, gilt es den Willen eines urteilsfähigen Kindes zu respektieren und einen Eingriff ggf. zu verschieben. Die Anwendung körperlichen Zwangs sollte auch bei Kleinkindern nur wohl- überlegt erfolgen und - dem Kind sowie den Eltern - vorher so gut wie möglich kommuniziert werden.}

\section{Korrespondenzadresse \\ Dr. A. Zutter}

Abteilung für Pädiatrische Intensivbehandlung (APIB), Universitätsklinik für Kinderheilkunde, Inselspital

3010 Bern, Schweiz

Andreas.Zutter@insel.ch

Interessenkonflikt. Der korrespondierende Autor gibt an, dass kein Interessenkonflikt besteht.

\section{Literatur}

1. Hearst D (2009) The runaway child. Managing anticipatory fear, resistance and distress in children undergoing surgery. Pediatr Anesth 19:1014-1016

2. Watson A, Visram A (2003) Children's preoperative anxiety and postoperative behaviour. Pediatr Anesth 13:188-204

3. Kain ZN, Wang SM, Mayes LC et al (1999) Distress during induction of anesthesia and behavioural outcomes. Anesth Analg 88:1042-1047

4. Kain ZN, Mayes LC, O'Connor TZ, Cicchetti DV (1996) Preoperative anxiety in children, predictors and outcomes. Arch Pediatr Adolesc Med 150:1238-1245

5. Bevan JC, Johnston C, Haig MJ et al (1990) Preoperative parental anxiety predicts behaviour and emotional responses to induction of anaesthesia in children. Can J Anaesth 37:177-182

6. Holm-Knudsen RJ, Carlin JB, McKenzie IM (1998) Distress at induction of anaesthesia in children. A survey of incidence, associated factors and recovery characteristics. Paediatr Anaesth 8:383-392

7. Kotiniemi LH, Ryhanen PT, Moilanen IK (1997) Behavioural changes in children following daycase surgery: a 4 week follow-up of 551 children. Anesthesia 52:970-976

8. Kotiniemi LH, Ryhanen PT (1996) Behavioural changes in children's memories after intravenous, inhalational and rectal induction of anesthesia. Pediatr Anesth 6:201-207

9. Kain ZN (2001) Premedication and parental presence. Curr Opin Anesthesiol 14:331-337

10. Kain ZN, Mayes CM, Caramico LA et al (1996) Parental presence during induction of anesthesia. A randomized controlled trial. Anesthesiology 84:1060-1067

11. Messeri A, Caprilli S, Busoni P (2004) Anaesthesia induction in children: a pyschological evaluation of the efficiency of parents' presence. Pediatr Anesth 14:551-556

12. Kain ZN, Mayes CM, Wang SM et al (1998) Parental presence during induction of anesthesia vs. sedative premedication: which intervention is more effective? Anesthesiology 89:1147-1156

13. Litman RS, Berger AA, Chhibber A (1996) An evaluation of preoperative anxiety in a population of infants and children undergoing ambulatory surgery. Pediatr Anesth 6:443-447

14. Kain ZN, Mayes LC, Wang SM et al (2000) Parental presence and sedative premedicant for children undergoing surgery: a hierarchical study. Anesthesiology 92:939-946

15. Machotta A (2010) Das unkooperative Kind in der Einleitung. Anasthesiol Intensivmed Notfallmed Schmerzther 45:378-382

16. Fortier MA, MacLaren Chorney J, Zisk Rony RY et al (2009) Children's desire for periopertive information. Anesth Analg 109:1085-1090

17. Kain ZN, Mayes LC, Caramico LA (1996) Preoperative preparation in children: a cross-sectional study. J Clin Anesth 8:508-514

18. Yip P, Middleton P, Cyna AM, Carlyle AV (2009) Non-pharmacological interventions for assisting the induction of anesthesia in children. Cochrane Database Syst Rev 8:CD006447

19. Wang SM, Maranets I, Weinberg ME et al (2001) Parental auricular acupuncture as an adjunct for parental presence during induction of anesthesia. Anesthesiology 100:1399-1404

20. Vagnoli L, Caprilli S, Robiglio A et al (2005) Clown doctors as a treatment for preoperative anxiety in children: a randomized, prospective study. Pediatrics 116:563-567
21. Calipel S, Lucas-Polomeni MM, Wodey E et al (2005) Premedication in children: hypnosis versus midazolam. Pediatr Anesth 15:275-281

22. Patel A, Schieble T, Davidson M et al (2006) Distraction with a hand-held video game reduces pediatric preoperative anxiety. Pediatr Anesth 16:1019-1027

23. Low DK, Pittaway AP (2008) The, iPhone' induction - a novel use for the Apple iPhone. Pediatr Anesth 18:573-574

24. Rosenbaum A, Kain ZN, Larsson P et al (2009) The place of premedication in pediatric practice. Pediatr Anesth 19:817-828

25. Bozkurt P (2007) Premedication of the pediatric patient: anesthesia for the uncooperative child. Curr Opin Anesthesiol 20:211-215

26. Coté CJ, Cohen IT, Suresh S et al (2002) A comparison of three doses of a commercially prepared oral midazolam syrup in children. Anesth Analg 94:1-3

27. Lönnqvist P, Habre W (2005) Midazolam as premedication. Is the emperor naked or just half-dressed? Pediatr Anesth 15:263-265

28. Kain ZN, Mayes LC, Wang SM et al (1999) Postoperative behavioural outcomes in children: effects of sedative premedication. Anesthesiology 90:758-765

29. Cox RG, Nemish U, Ewen A et al (2006) Evidencebased clinical update: does premedication with oral midazolam lead to improved behavioural outcomes in children? Can J Anesth 53:1213-1219

30. Lin C, Durieux ME (2005) Ketamine and kids: an update. Pediatr Anesth 15:91-97

31. Koinig H, Marhofer P (2003) S(+)ketamine in pediatric anesthesia. Pediatr Anesth 13:185-187

32. Gutstein HB, Johnson KL, Heard MB et al (1992) Oral ketamine preanesthetic medication in children. Anesthesiology 76:28-33

33. Warner DL, Cabaret J, Velling D (1995) Ketamine plus midazolam, a most effective pediatric oral premedicant. Pediatr Anesth 5:293-295

34. Holm-Knudsen R, Sjogren P, Laub M (1990) Midazolam und Ketamin zur rektalen Prämedikation und Narkoseeinleitung bei Kindern. Anaesthesist 39:255-257

35. Bergendahl $H$, Lönnqvist PA, Eksborg S (2006) Clonidine in paediatric anaesthesia: review of the literature and comparison with benzodiazepines for premedication. Acta Anaesthesiol Scand 50:135-143

36. Lewis I, Burke C, Voepel-Lewis T et al (2007) Children who refuse anesthesia or sedation: a survey of anesthesiologists. Pediatr Anesth 17:1134-1142

37. Committee on Bioethics (1995) Informed consent, parental permission and assent in pediatric practice. Pediatrics 95:314-317

38. Dare T (2009) Parental rights and medical decisions. Pediatr Anesth 19:947-952

39. Schweizerische Akademie der Medizinischen Wissenschaften: Recht der Patientinnen und Patienten auf Selbstbestimmung. Medizinisch-ethische Grundsätze der SAMW. http://www.samw.ch/de/ Ethik/Richtlinien/Aktuell-gueltige-Richtlinien.html

40. Deklaration von Ottawa zum Recht des Kindes auf gesundheitliche Versorgung. http://www.bundesaerztekammer.de/Patienten/Patientenrechte. Englische Version: Declaration of Ottawa on the Right of the Child to Health Care 1998. http://www. wma.net/en/40news/20archives/1998/1998_06/ index.html 